\title{
PulmoBind, an Adrenomedullin-Based Molecular Lung Imaging Tool
}

\author{
Myriam Létourneau*1, Quang Trinh Nguyen*2, François Harel ${ }^{2,3}$, Alain Fournier ${ }^{1}$, and Jocelyn Dupuis ${ }^{2,4}$ \\ ${ }^{1}$ Laboratoire d'Études Moléculaires et Pharmacologiques des Peptides, INRS-Institut Armand-Frappier, Laval, Quebec, Canada; \\ ${ }^{2}$ Research Centre, Montreal Heart Institute, Montreal, Quebec, Canada; ${ }^{3}$ Department of Radiology, Université de Montréal, \\ Montreal, Quebec, Canada; and ${ }^{4}$ Department of Medicine, Université de Montréal, Montreal, Quebec, Canada
}

\begin{abstract}
Previous studies showed that adrenomedullin (AM) could be a promising agent for molecular imaging of the pulmonary circulation, with abundant specific binding sites at the pulmonary vascular endothelium. The purpose of this work was to design an AM-based compound that encompasses the desired imaging properties without posing safety issues for clinical applications. Methods: AM analogs were synthesized through solid-phase peptide synthesis. They were evaluated for ${ }^{99 \mathrm{~m} T c}$ labeling efficiency and in vivo lung uptake. Biodistribution and hemodynamic characteristics of the lead compound were determined in anesthetized dogs as well as by a dosimetric analysis. Lung perfusion was evaluated in the monocrotaline model of pulmonary arterial hypertension in rats. Results: $\mathrm{A}$ cyclic AM (residues 22-52) analog encompassing a polyethylene glycol spacer and a tetrapeptide chelating moiety was found to possess the desired characteristics, with $90.7 \% \pm 0.3 \%$ (mean \pm SD) labeling efficiency, $40 \%$ lung uptake at $10 \mathrm{~min}$ after injection, and a favorable safety profile. Lung uptake of the ${ }^{99 m}$ Tc-labeled compound was markedly reduced in rats with pulmonary arterial hypertension. Conclusion: This lead compound could be a suitable clinical imaging agent for the molecular diagnosis of disorders of the pulmonary circulation.
\end{abstract}

Key Words: lung; molecular imaging; adrenomedullin; nuclear medicine; pulmonary hypertension

J Nucl Med 2013; 54:1789-1796

DOI: 10.2967/jnumed.112.118984

$\mathbf{P}$ ulmonary embolism is a potentially lethal lung perfusion defect that commonly develops in patients with deep vein thrombosis (1). Pulmonary hypertension, a disorder with various etiologies, leads - in its most severe forms - to right ventricular failure and death (2). Diagnosis of these pathologies is challenging because patients often have a nonspecific symptom, shortness of breath. At present, nuclear imaging of the pulmonary circulation can be achieved through the use of ${ }^{99 \mathrm{~m}} \mathrm{Tc}$-albumin macroaggregates, particles that are trapped in the microvasculature because of their size $(10-150 \mu \mathrm{m})$ in proportion to the regional blood flow (3). However,

Received Dec. 21, 2012; revision accepted Apr. 12, 2013.

For correspondence contact: Jocelyn Dupuis, Research Centre, Montreal Heart Institute, 5000 Belanger St., Montreal, Quebec, Canada H1T 1 C8.

E-mail: jocelyn.dupuis@bellnet.ca

${ }^{*}$ Contributed equally to this work.

Published online Aug. 15, 2013.

COPYRIGHT (C) 2013 by the Society of Nuclear Medicine and Molecular Imaging, Inc. this technique lacks the ability to detect functional perfusion defects, and its ability to detect small-blood-vessel blockade is limited. Moreover, this tracer blocks pulmonary blood vessels in subjects with an already compromised pulmonary circulation and increases the potential risk for infections because it is prepared from human albumin.

We recently showed that adrenomedullin (AM) is a promising molecular lung imaging tool for the diagnosis of pathologies such as pulmonary embolism and pulmonary hypertension $(4,5)$. AM, a peptide from the calcitonin family, allows specific lung imaging through binding with its specific receptor, AM1 (CRLR/RAMP2), which is expressed at a high density in pulmonary vessels $(6,7)$. Enhanced imaging efficiency can be associated with cell internalization after activation of the receptor by its agonist $(8,9)$, but this process is also correlated with inherent biologic activity. Conversely, antagonists do not evoke cellular activation in receptor binding but are able to bind with uniform affinity to the total pool of receptors $(10,11)$.

AM, a 52-amino-acid multifunctional regulatory peptide, is widely distributed and acts mainly in the cardiovascular system, for which it is classically described as a vasodilator (12). Previous structureactivity relationship studies demonstrated that truncating the $\mathrm{N}$ terminal stretch while maintaining the cyclic structure formed by a disulfide bridge between residues 16 and 21 generated agonists, such as AM with residues 13-52 [AM(13-52)] and AM(16-52), with affinities similar to that of the full-length peptide (13). Further truncation at the $\mathrm{N}$ terminus to remove the ring structure yielded the only fully characterized human AM antagonist, that is, AM (22-52); in contrast, removal of the C-terminal amino acid greatly diminished binding affinity and peptide activity $(13,14)$. Interestingly, analogs composed only of the ring structure, that is, AM (15-22) and AM(16-21), increased systemic arterial blood pressure (15), but $\mathrm{AM}(1-25)$ and $\mathrm{AM}(1-40)$ were unable to displace ${ }^{125} \mathrm{I}-\mathrm{AM}$ in a dog lung homogenate binding experiment (6). Moreover, linearization of the full-length peptide resulted in a loss of affinity $(6,13)$. Finally, although AM is a long peptide, the incorporation of a radionuclide can affect its binding ability; therefore, AMderived nuclear imaging tools should favor radiolabeling at the $\mathrm{N}$ terminus of the peptide (16).

$\mathrm{AM}(22-52)$ is an antagonist that has 10 times less affinity for its receptors than $\mathrm{AM}(13-52)$ in competition displacement experiments but that has higher selectivity for AM1 than for AM2 (CRLR/ RAMP3) $(6,14)$. Thus, this peptide appeared to be a good starting point for generating new AM antagonists suitable for lung nuclear imaging. Such analogs would enable pulmonary microcirculation imaging without any side effects on blood pressure. Furthermore, and as pointed out for AM, such molecules not only would be 
valuable for detecting perfusion defects but also could allow the detection of early endothelium-related deficiencies, such as pulmonary hypertension.

In this article, we describe the design, synthesis, and characterization of PulmoBind, an AM-based analog for pulmonary circulation imaging. This new molecular lung imaging tool has good binding affinity, high ${ }^{99 \mathrm{~m}} \mathrm{Tc}$ labeling efficiency, and significant lung uptake even after $1 \mathrm{~h}$ without any effect on mean arterial pressure (MAP).

This molecule could make it possible to detect not only largepulmonary-vessel blockade, such as a pulmonary embolism, but also anatomic and, potentially, functional pulmonary microcirculatory disorders caused by various forms of pulmonary hypertension. Thus, PulmoBind could provide an efficient, safe, and noninvasive method for probing the status of the pulmonary circulation.

\section{MATERIALS AND METHODS}

All animal experimental procedures were performed in accordance with regulations and ethical guidelines of the Canadian Council for the Care of Laboratory Animals and received approval from the Animal Ethics and Research Committee of the Montreal Heart Institute.

\section{AM Derivative Synthesis and Purification}

AM-derived peptides were synthesized and purified as previously described (6). The Fmoc-D-(polyethylene glycol) ${ }_{4}-\mathrm{COOH}$ acid used as a spacer in the peptide synthesis was purchased from Quanta Biodesign Ltd. Disulfide bond formation to yield cyclic derivatives was performed by overnight air oxidation after dissolution of the linear peptide in an aqueous solution ( $\mathrm{pH} 8.8$ ) at a concentration of $0.1 \mathrm{mg} / \mathrm{mL}$. Peptides were purified by reversed-phase high-performance liquid chromatography (HPLC). The amino acid sequences of the synthesized peptides are shown in Table 1, along with the purity of the final products and the molecular masses, as evaluated by matrix-assisted laser desorption ionization-time-of-flight mass spectrometry (Voyager DE; Applied Biosystems).

\section{9mTc Labeling}

Linear AM was radiolabeled through its cysteine residues as previously described (5). For AM analogs containing a chelating moiety, $2.29 \mathrm{nmol}$ of lyophilized peptide were suspended in $20 \mu \mathrm{L}$ of $1 \mathrm{M}$ acetate buffer (pH 5.5), to which $200 \mu \mathrm{L}$ of $0.1 \mathrm{M} \mathrm{Na}_{3} \mathrm{PO}_{4}$ (pH 12) and
$31.25 \mu \mathrm{L}$ of freshly prepared $\mathrm{SnCl}_{2}$ solution $(0.8 \mathrm{mg} / \mathrm{mL}$, in $0.05 \mathrm{M}$ $\mathrm{HCl}$ ) were added. Immediately after dissolution, sterile and freshly prepared (on a daily basis) $\mathrm{Na}^{99 \mathrm{~m}} \mathrm{TcO}_{4}(185-555 \mathrm{MBq})$ in a sodium chloride injection solution (U.S. Pharmacopeia) was added to the mixture. The mixture was kept at room temperature for $15 \mathrm{~min}$, and $1 \mathrm{~mL}$ of $0.1 \mathrm{M} \mathrm{NaH}_{2} \mathrm{PO}_{4}(\mathrm{pH} 4.5$ ) was finally added. Radiochemical purity and labeling efficiency were assessed by instant thin-layer chromatography (ITLC) on silica gel-impregnated glass fiber filter paper with acetone for the dosage of free ${ }^{99 \mathrm{~m}} \mathrm{Tc}$ and $37.5 \%(\mathrm{v} / \mathrm{v})$ butanol$7.5 \%(\mathrm{v} / \mathrm{v})$ acetic acid-30\% (v/v) pyridine in water to discriminate 99m Tc-colloids (bottom) from radiolabeled peptides (top).

For purifying ${ }^{99 \mathrm{~m}} \mathrm{Tc}$-labeled AM derivatives, the radiolabeling reaction mixture was injected into a 1-mL (100-mg) $\mathrm{C}_{18}$ SepPak (Waters) cartridge. The cartridge was then washed with $3 \mathrm{~mL}$ of $1 \mathrm{mM}$ hydrochloric acid and eluted with $3 \mathrm{~mL}$ of a $50 \%(\mathrm{v} / \mathrm{v})$ solution of ethanol and water. Fractions of $0.5 \mathrm{~mL}$ were collected into sterile polypropylene tubes. Fractions with the highest counts were pooled, and $200 \mu \mathrm{L}$ of $10 \times$ concentrated phosphate-buffered saline $(\mathrm{pH} 7.4)$ were added for stabilization.

\section{In Vivo Biodistribution and Lung Uptake}

Mongrel dogs (20-30 kg) were anesthetized by intravenous injection of sodium pentobarbital $(50 \mathrm{mg} / \mathrm{kg})$, intubated, and mechanically ventilated. Purified ${ }^{99 \mathrm{~m}} \mathrm{Tc}$-labeled AM derivatives $(185 \mathrm{MBq})$ were injected intravenously via the jugular vein through a 3-way stopcock 18 -French catheter for lung uptake evaluation. In vivo whole-body biodistribution of radiolabeled peptides was evaluated by use of a dualhead $\gamma$-camera (Siemens) equipped with a low-energy parallel-hole collimator. Dynamic acquisitions were recorded for a 30-min period, and static whole-body scans were obtained at 30, 60, and $120 \mathrm{~min}$ after ${ }^{99 \mathrm{~m}} \mathrm{Tc}$-labeled peptide injection at a scan speed of $10 \mathrm{~cm} / \mathrm{min}$. Dynamic and static acquisitions were evaluated with MATLAB version 7.01 image analysis tool software. Data correction was applied for radioactive decay, table adjustment (dorsal images only), geometric mean, and organ attenuation based on the transmission factor. Results were expressed as a percentage of the total radioactivity injected.

\section{Binding Assays}

The binding ability of PulmoBind was evaluated with dog lung homogenates prepared as previously described (6). In brief, lungs were diced, homogenized, and centrifuged at $49,000 \mathrm{~g}$ for $20 \mathrm{~min}$ at $4^{\circ} \mathrm{C}$. Pellets were washed twice, and nonhomogenized tissue was removed by gentle centrifugation $(500 \mathrm{~g} ; 5 \mathrm{~min})$. Aliquots of the supernatant

TABLE 1

Human AM (hAM) and Peptide Analogs Synthesized

\begin{tabular}{|c|c|c|c|c|}
\hline Compound & Sequence & $\begin{array}{l}\text { Purity } \\
(\%)\end{array}$ & $\begin{array}{l}\text { Theoretic } \\
\text { molecular mass }\end{array}$ & $\begin{array}{l}\text { Observed } \\
\text { molecular mass }\end{array}$ \\
\hline hAM & $\begin{array}{l}\text { Tyr-Arg-Gln-Ser-Met-Asn-Asn-Phe-Gln-Gly-Leu-Arg-Ser-Phe-Gly- } \\
\text { Cys-Arg-Phe-Gly-Thr-Cys-Thr-Val-Gln-Lys-Leu-Ala-His-Gln-lle-Tyr- } \\
\text { Gln-Phe-Thr-Asp-Lys-Asp-Lys-Asp-Asn-Val-Ala-Pro-Arg-Ser-Lys-lle- } \\
\text { Ser-Pro-Gln-Gly-Tyr }\end{array}$ & 98 & $6,028.82$ & $6,028.18$ \\
\hline hAM(13-52) & $\begin{array}{l}\text { Ser-Phe-Gly-Cys-Arg-Phe-Gly-Thr-Cys-Thr-Val-Gln-Lys-Leu-Ala- } \\
\text { His-Gln-Ile-Tyr-Gln-Phe-Thr-Asp-Lys-Asp-Lys-Asp-Asn-Val-Ala- } \\
\text { Pro-Arg-Ser-Lys-Ile-Ser-Pro-Gln-Gly-Tyr }\end{array}$ & 97 & $4,533.15$ & $4,535.46$ \\
\hline hAM(22-52) & $\begin{array}{l}\text { Thr-Val-Gln-Lys-Leu-Ala-His-Gln-Ile-Tyr-Gln-Phe-Thr-Asp-Lys-Asp- } \\
\text { Lys-Asp-Asn-Val-Ala-Pro-Arg-Ser-Lys-lle-Ser-Pro-Gln-Gly-Tyr }\end{array}$ & 95 & $3,576.03$ & $3,578.58$ \\
\hline 1 & Gly-Gly-D-Ala-Gly-D-(PEG) $)_{4}$-hAM(22-52) & 92 & $4,064.16$ & $4,065.55$ \\
\hline 2 (PulmoBind) & Gly-Gly-D-Ala-Gly-Cys-D-(PEG) ${ }_{4}$-Cys-hAM(22-52) & 97 & $4,270.82$ & $4,271.71$ \\
\hline 3 & Gly-Gly-D-Ala-Gly-Cys(Acm)-D-(PEG)4-Cys(Acm)-hAM(22-52) & 96 & $4,412.82$ & $4,410.34$ \\
\hline
\end{tabular}




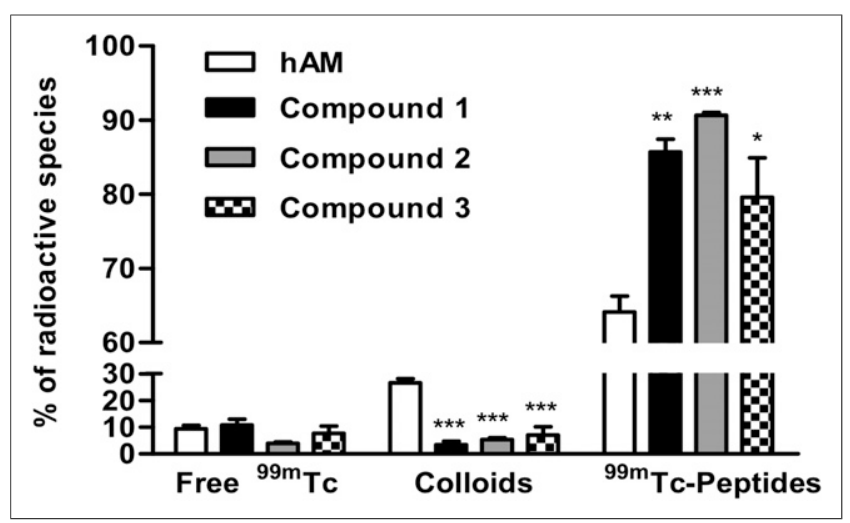

FIGURE 1. $99 \mathrm{~m}$ Tc labeling yields of $A M$ and its analogs. Reaction mixtures obtained after ${ }^{99 \mathrm{~m} T c}$ labeling procedure were analyzed by ITLC to determine relative proportions of radioactive species, that is, free ${ }^{99 m T c}$, colloids, and labeled peptides. Results are expressed as mean \pm SEM of 4 experiments, and significant differences from results for human AM (hAM) were determined with 1-way ANOVA (Tukey posttest). ${ }^{\star} P<0.05$. ${ }^{\star \star} P<0.01$. ${ }^{\star \star \star} P<0.001$.

were kept at $-80^{\circ} \mathrm{C}$ until used for binding assays. Binding experiments were based on previously described protocols $(6,16)$. The ${ }^{99 \mathrm{~m}} \mathrm{Tc}-$ labeled PulmoBind ligand was prepared as described earlier, and the 3 fractions containing the highest levels of radioactivity were pooled and used for assays. The concentration of this initial mixture was $1.527 \mu \mathrm{M}$, and the specific activity was $81 \mathrm{MBq} / \mathrm{mmol}$.

Competitive displacement experiments were carried out by incubating dog lung homogenates $(100 \mu \mathrm{L} ; 0.24 \mathrm{mg})$ for $90 \mathrm{~min}$ at room temperature in binding buffer $(50 \mathrm{mM}$ Tris, $100 \mathrm{mM} \mathrm{NaCl}, 4 \mathrm{mM}$ $\mathrm{MgCl}_{2}$, and $0.1 \%$ [w/v] bovine serum albumin; $\left.\mathrm{pH} 7.4\right)$ with various concentrations $\left(10^{-12}-10^{-5} \mathrm{M}\right)$ of either unlabeled PulmoBind or AM in the presence of $1 \mathrm{nM}{ }^{99} \mathrm{~m}$ Tc-labeled PulmoBind.

For saturation binding experiments, dog lung homogenates were incubated as described for competitive displacement experiments with increasing concentrations $(0.01-5 \mathrm{nM})$ of ${ }^{99 \mathrm{~m}} \mathrm{Tc}$-labeled PulmoBind in the absence (total binding) or presence of $1 \mu \mathrm{M}$ unlabeled ligand. Incubations were stopped by rapid filtration through glass fiber filter papers presoaked in $0.3 \%$ polyethyleneimine with a Millipore 1225 sampling vacuum manifold. After 3 washings, the activity in the papers was counted with an LKB Wallac 1272 automatic $\gamma$-counter.

\section{Hemodynamic Evaluation}

Mongrel dogs (male and female) were used to study cardiovascular effects induced by cumulative intravenous injections of human AM(152), the agonist human $\operatorname{AM}(13-52)$, the antagonist human $\operatorname{AM}(22-52)$, and PulmoBind. The heart rate of anesthetized and ventilated animals was monitored with cutaneous electrocardiographic leads, and a catheter installed in the right femoral artery was used to monitor blood pressure. Pulmonary artery pressure was evaluated with a Swan-Ganz catheter. In brief, the catheter was introduced through the jugular vein. From this entry site, it was threaded by fluoroscopy through the right atrium of the heart, through the right ventricle, and subsequently into the pulmonary artery until a wedge pressure tracing was noted. Next, the Swan-Ganz catheter balloon was deflated; deflation was confirmed by the reappearance of pulmonary artery pressure contours.

\section{Dosimetric Evaluation}

The PulmoBind tracer was injected into 7 dogs to estimate the mean biodistribution on whole-body scintigraphic images. Before peptide injection, a whole-body transmission scan was obtained with the $\gamma$-camera and a ${ }^{57} \mathrm{Co}$ flood source. The injection syringe was measured before injection under the camera. Immediately after intravenous injection, dynamic acquisitions were recorded for a 30-min period, and static whole-body scans were obtained 35, 60, and 120 min later at a scan speed of $10 \mathrm{~cm} / \mathrm{min}$. The empty syringe was measured again to determine residual activity.

The biodistribution into organs with significant uptake was evaluated with MATLAB version 7.01 image analysis tool software. Dynamic and whole-body images were first corrected for radioactive decay. Regions of interest were drawn on each organ in anterior and posterior views and on the transmission map. The geometric mean of the activity in each organ was determined and then corrected for average attenuation over the organ. Syringe image data were used to convert the results to percentages of the total radioactivity injected.

These animal studies with dogs were performed to obtain a first approximation of the expected radiation dose absorbed in human subjects. Despite differences between animal and human pharmacokinetic behaviors, an extrapolation process was used to estimate human dosimetry from the calculated dog biodistribution. To compensate for the physiologic time difference between species, allometric scaling was applied to the biodistribution curves, assuming human physiologic time to be 1.19 times slower than canine physiologic time (fourth root of the mass ratio) (17). Individual organ and whole-body time-activity curves were fitted with OLINDA software version 1.0 (18). Target organ absorbed radiation doses were calculated for a 73.7-kg standard man model and a 56.9-kg standard woman model with the same software.

\section{Lung Perfusion in Pulmonary Arterial Hypertension}

Male Sprague-Dawley rats weighting 200-225 g received a $0.5-\mathrm{mL}$ intraperitoneal injection of either $0.9 \%$ saline $(n=4)$ or monocrotaline at $60 \mathrm{mg} / \mathrm{kg}(n=4)$. Five weeks later, rats were anesthetized for hemodynamic measurements and nuclear medicine experiments with PulmoBind as previously described (4).

\section{Statistics}

Statistical analyses were assessed with Prism 4.0 software (Graph$\mathrm{Pad}$ ) and an unpaired Student $t$ test or a 1-way ANOVA.

\section{RESULTS}

\section{Synthesis}

AM analogs were designed in a simple and straightforward manner based on our previous results with $\mathrm{AM}$ as a ${ }^{99 \mathrm{~m}} \mathrm{Tc}-\mathrm{labeled}$ imaging agent and known structure-activity relationships. In fact,

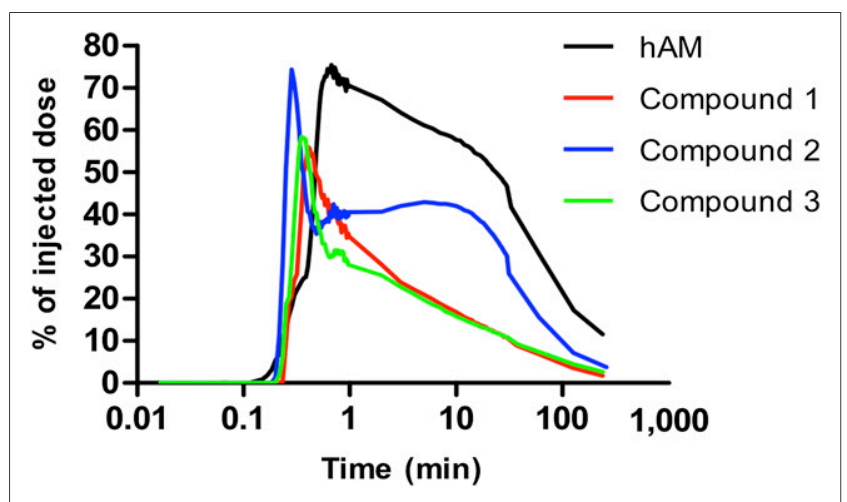

FIGURE 2. In vivo lung uptake of ${ }^{99 m}$ Tc-labeled human AM (hAM) and analogs in dogs. Dynamic acquisitions were recorded with dual-head camera at scan speed of $10 \mathrm{~cm} / \mathrm{min}$ over 2-h period after injection of $185 \mathrm{MBq}$ of purified ${ }^{99 \mathrm{~m} T c-l a b e l e d}$ compound. 
linear AM had been directly labeled through its free cysteine residues and purified with a SepPak cartridge to obtain a final product with a high radiochemical purity ( $\geq 95 \%)(5)$. However, the initial yield of ${ }^{99 \mathrm{~m}} \mathrm{Tc}$-labeled AM, that is, before the purification step, was only about $65 \%$ (Fig. 1). Therefore, we added a chelating moiety to trap the radioisotope. A simple tetrapeptide having ${ }^{99 \mathrm{~m}} \mathrm{Tc}$ chelating properties, Gly-Gly-D-Ala-Gly (19), was used. Thus, compound 1 was created by complexing the antagonist $\mathrm{AM}(22-$ 52) to this tetrapeptide ${ }^{99 \mathrm{~m}} \mathrm{Tc}$ chelating moiety through a molecular linker, the heterobifunctional polyethylene glycol derivative FmocD-(polyethylene glycol) ${ }_{4}-\mathrm{COOH}$. Two cysteine residues were introduced into the AM analog construction to generate cyclic compound 2. Finally, compound 3 was designed with Cys acetamidomethyl $(\mathrm{Acm})$ residues, which cannot form a disulfide bridge because their sulfhydryl moieties are blocked by an Acm group, to verify the impact of cyclization on lung uptake and the influence of cysteine residues in the labeling process.

All peptide syntheses yielded only 1 major product that was isolated by reversed-phase HPLC and confirmed to be the desired AM analog by matrix-assisted laser desorption ionization-time-of-flight mass spectrometry. Formation of the disulfide bond was monitored by analytic reversed-phase HPLC, the cyclized peptide having a shorter retention time than its linear counterpart (results not shown).

\section{9mTc Labeling}

As expected, the introduction of a chelating moiety greatly improved the ${ }^{99 \mathrm{~m}} \mathrm{Tc}$ labeling yield of AM analogs over that of linear AM (Fig. 1). In fact, the labeling of AM through its free cysteine residues resulted, on average, in $65 \%{ }^{99 \mathrm{~m}} \mathrm{Tc}$-labeled AM, whereas the labeling yield was higher than $80 \%$ when the tetrapeptide was used as the ${ }^{99 \mathrm{~m}} \mathrm{Tc}$ chelator. Moreover, the labeling yield obtained with compounds 1,2 , and 3 was not markedly affected by the nature of the peptide, although yields varied slightly with compound 2, which had the greatest ability to retain ${ }^{99 \mathrm{~m}} \mathrm{Tc}$ (Fig. 1).

\section{In Vivo Biodistribution and Lung Uptake}

Because satisfying ${ }^{99 \mathrm{~m}} \mathrm{Tc}$ labeling yields were obtained with the 3 AM analogs, their lung uptake ability was evaluated in anesthetized dogs. Purified labeled compound 1, 2, or 3 (radiochemical purity of $\geq 95 \%$, as evaluated by ITLC) was injected intravenously, and a lung dynamic acquisition was performed. All 3 compounds showed reduced lung uptake compared with that of AM (Fig. 2). However, compound 2 had a lung first pass comparable to that observed with AM ( $\sim 75 \%$ of the injected dose), rapidly followed in the first minute by a $40 \%$ uptake plateau maintained for up to $1 \mathrm{~h}$ (Fig. 2). For compounds 1 and 3, initial lung uptake was considerably reduced ( $\sim 55 \%$ of the injected dose), and the compounds were continuously cleared from the pulmonary circulation (Fig. 2).

Interestingly, at $30 \mathrm{~min}$ after injection, heart uptake of compound 2 was low $(3.1 \% \pm 0.3 \%$ [mean $\pm \mathrm{SD}])$ - even lower than that of AM $(4.9 \% \pm 1.0 \%)$-although not significantly different, an important asset for obtaining good lung imaging (Fig. 3). Moreover, liver uptake of compound 2 was comparable to that of AM. Thus, with liver uptake of less than $10 \%(8.2 \% \pm 0.8 \%)$ of the injected dose, lung uptake at least 5 times as high as heart uptake, and lung retention lasting up to $1 \mathrm{~h}$, compound 2 appeared to be a good candidate for lung imaging (Fig. 4). Further analyses were performed to characterize this compound, renamed PulmoBind.

\section{Binding Assays}

For further characterization of this new lung imaging agent, binding assays were performed with dog lung homogenates and ${ }^{99 m}$ Tc-PulmoBind as the tracer. Saturation experiments demonstrated that PulmoBind bound to specific binding sites in the dog lung at a density of $2,317 \pm 320 \mathrm{fmol} / \mathrm{mg}$ (maximum binding potential $\left[\mathrm{B}_{\max }\right]$ ), with an affinity in the nanomolar range (dissociation constant $\left[\mathrm{K}_{\mathrm{d}}\right], 2.6 \pm 0.8 \mathrm{nM}$ ) (Figs. 5A and 5B). These values indicated that PulmoBind could occupy more binding sites than AM in the dog lung but at a lower affinity. Indeed, a similar set of experiments was performed with AM and the same preparation (16), thus allowing a comparison. It was found that AM bound specific binding sites at a density of 1,222 $\pm 148 \mathrm{fmol} / \mathrm{mg}$, with a $\mathrm{K}_{\mathrm{d}}$ of $0.17 \pm 0.07 \mathrm{nM}$.

For verification that PulmoBind had a binding site different from that of AM in the dog lung, competitive displacement experiments were performed. With either unlabeled AM or PulmoBind, displacement curves were statistically indistinguishable, suggesting that $\mathrm{AM}$ and PulmoBind competed for the same binding site in the dog lung (Fig. 5C).

\section{Hemodynamic Evaluation}

AM is a known vasodilator. When injected intravenously into anesthetized dogs, increasing doses of AM produced a drop in mean arterial blood pressure (MAP) that was accompanied by an elevation in heart rate (Fig. 6A). Similarly, the truncated AM agonist, human $\mathrm{AM}(13-52)$, generated decreases in MAP proportional to the increasing doses of peptide injected. It was noteworthy that the accompanying

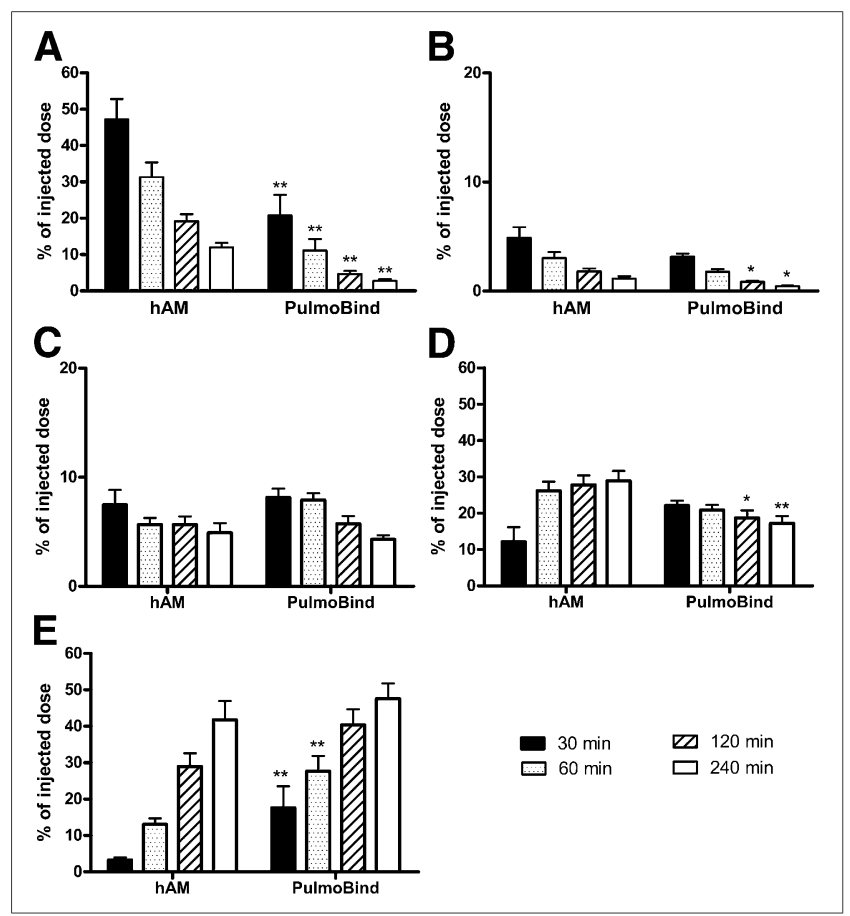

FIGURE 3. In vivo biodistribution of ${ }^{99 m}$ Tc-labeled human AM (hAM) and PulmoBind. Purified ${ }^{99 m}$ Tc-labeled peptides (185 MBq) were injected intravenously into dogs, and then static evaluation was performed with dual-head $\gamma$-camera and MATLAB software to analyze data. Graphs represent data recorded for lungs $(A)$, heart $(B)$, liver (C), kidneys (D), and bladder (E) in 7 dogs for hAM and 5 dogs for PulmoBind. Significant differences were determined with unpaired $t$ test for PulmoBind vs. hAM. ${ }^{\star} P<0.05$. ${ }^{\star \star} P<0.01$. 


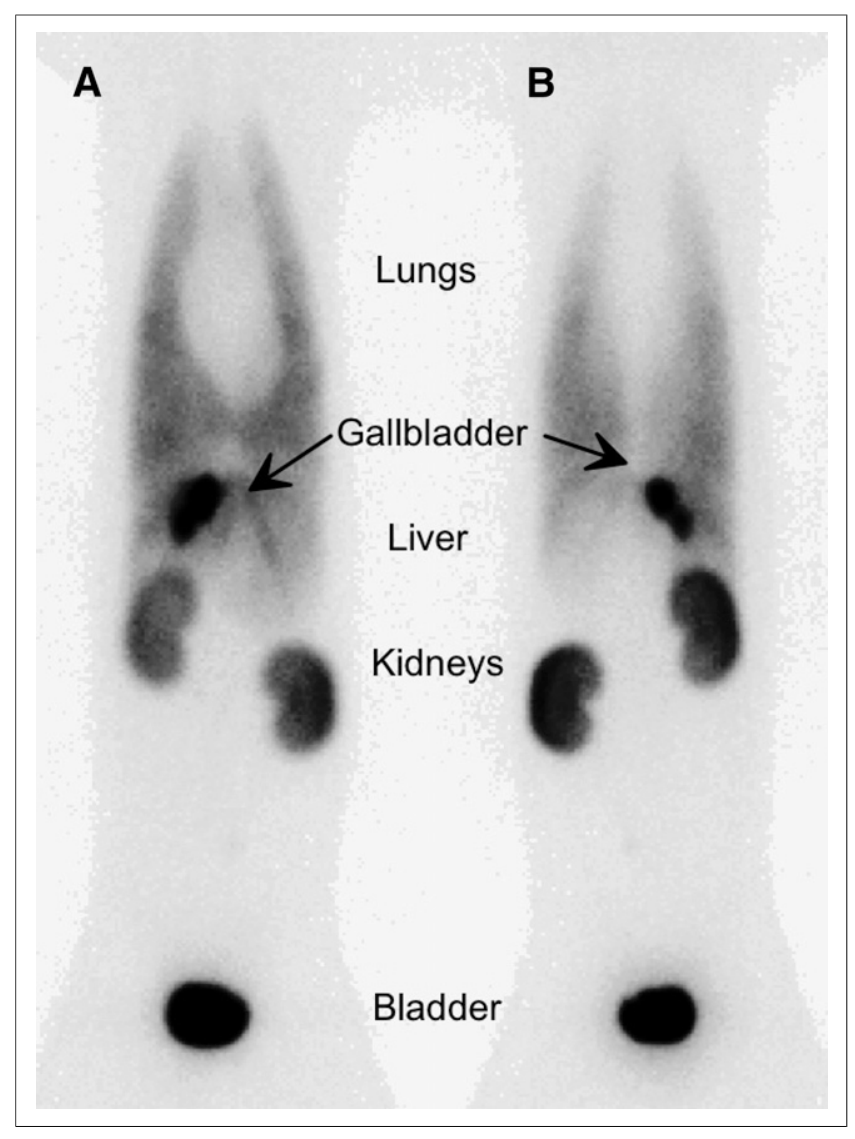

FIGURE 4. Dog imaging with ${ }^{99 m}$ Tc-PulmoBind 60 min after intravenous injection of $185 \mathrm{MBq}$. (A) Anterior view. (B) Posterior view.

increase in heart rate was less pronounced than that observed with $\mathrm{AM}$, although the decreases in MAP were similar (Fig. 6B). When cumulative doses of human AM(22-52), a specific AM antagonist, were injected, no changes in MAP or heart rate were observed, even at a dose 50 times the initial injection (Fig. 6C). In a similar manner, cumulative intravenous injections of PulmoBind, which is derived from the latter antagonist, did not produce significant variations in MAP and heart rate, suggesting that this new lung imaging agent also acts as an antagonist (Fig. 6D). Finally, mean pulmonary artery pressure did not vary significantly after injections of human $\mathrm{AM}(22-52)$ and PulmoBind and was within expected values.

\section{Dosimetric Analysis}

A dosimetric analysis of PulmoBind is shown in Table 2. This analysis was extrapolated to humans from the biodistribution obtained in dogs. The analysis revealed a favorable profile, with rapid elimination of the tracer into urine and the digestive tract, with the kidneys receiving the highest radiation dose $(0.034 \mathrm{mGy} / \mathrm{MBq})$ (Table 2). The total effective doses were determined (under allometric scaling) to be 0.0075 and $0.0094 \mathrm{mSv} / \mathrm{MBq}$ for men and women, respectively, after $2 \mathrm{~h}$ (Table 3 ).

\section{Pulmonary Arterial Hypertension Model}

As shown in Figure 7, the in vivo biodistribution revealed markedly reduced lung uptake of PulmoBind, from $12 \pm 2$ percentage injected dose in control rats to $4 \pm 1$ percentage injected dose in rats with pulmonary arterial hypertension $(P<0.001)$.

\section{DISCUSSION}

Nuclear medicine offers clinicians novel avenues for the diagnosis and therapy of various pathologic conditions with noninvasive and rapid techniques. In fact, the success of nuclear medicine relies on the effectiveness of radiopharmaceutical compounds. These compounds, consisting of a target-specific moiety and a radionuclide, must be well designed and finely tuned to attain the desired results for a particular purpose. Because of their inherent specificity, antibodies are biomolecules of interest for generating pharmaceutical compounds. However, their poor pharmacokinetics and their tendency to evoke an immunogenic response (20) limit their effectiveness. On the other hand, peptides, which are also highly specific biomolecules, proved to be useful targeting moieties for generating therapeutic or imaging agents $(21,22)$ with the advantages of being practically devoid of immunogenicity and showing favorable pharmacokinetics (rapid clearance from blood). Moreover, they are quite flexible in terms of chemical modification, allowing radiolabeling (23).

We previously showed that the linear AM peptide, once labeled with ${ }^{99 m} \mathrm{Tc}$, was an attractive lung imaging agent for the diagnosis of pulmonary embolism (5). Lung perfusion scintigraphy with ${ }^{99 \mathrm{~m}} \mathrm{Tc}-$ labeled macroaggregates of albumin ( $\left.{ }^{99 \mathrm{~m} T c-M A A}\right)$ is generally con-
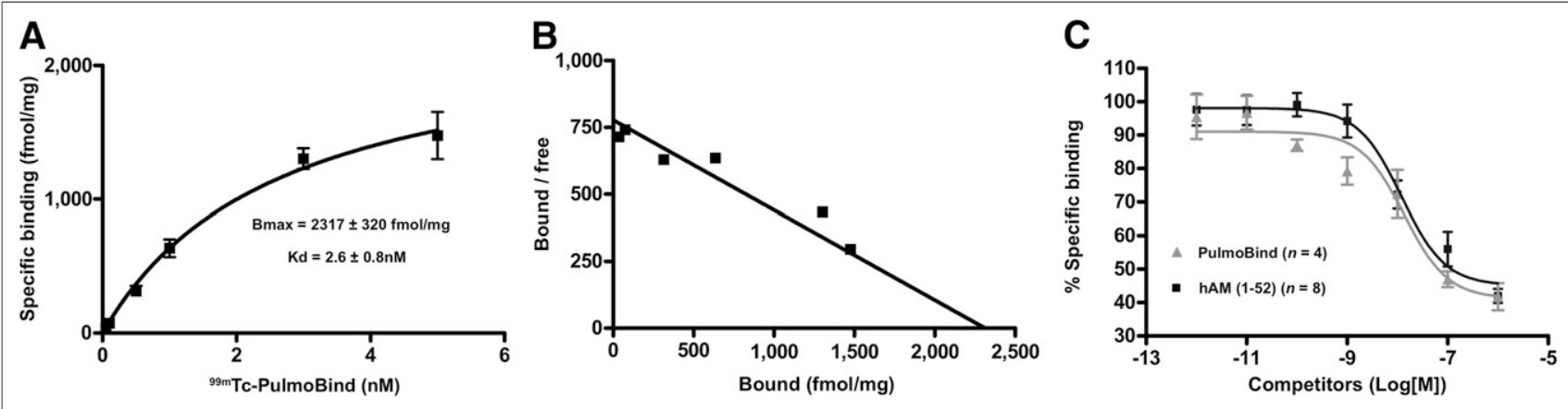

FIGURE 5. Characterization of binding of PulmoBind to dog lung homogenates. (A) Saturation of specific binding sites evaluated with increasing concentrations of ${ }^{99 \mathrm{~m} T c-P u l m o B i n d}$. (B) Scatchard plot derived from saturation experiment data. (C) Competitive displacement of bound ${ }^{99 m T c-P u l m o B i n d ~ w i t h ~ i n c r e a s i n g ~ c o n c e n t r a t i o n s ~ o f ~ u n l a b e l e d ~ P u l m o B i n d ~ o r ~ h u m a n ~ A M ~(h A M) . ~ R e s u l t s ~ a r e ~ e x p r e s s e d ~ a s ~ p e r-~}$ centage of specific binding, which was obtained by subtracting nonspecific binding (in presence of $1 \mu \mathrm{M}$ unlabeled ligand) from total binding (labeled peptide alone). 


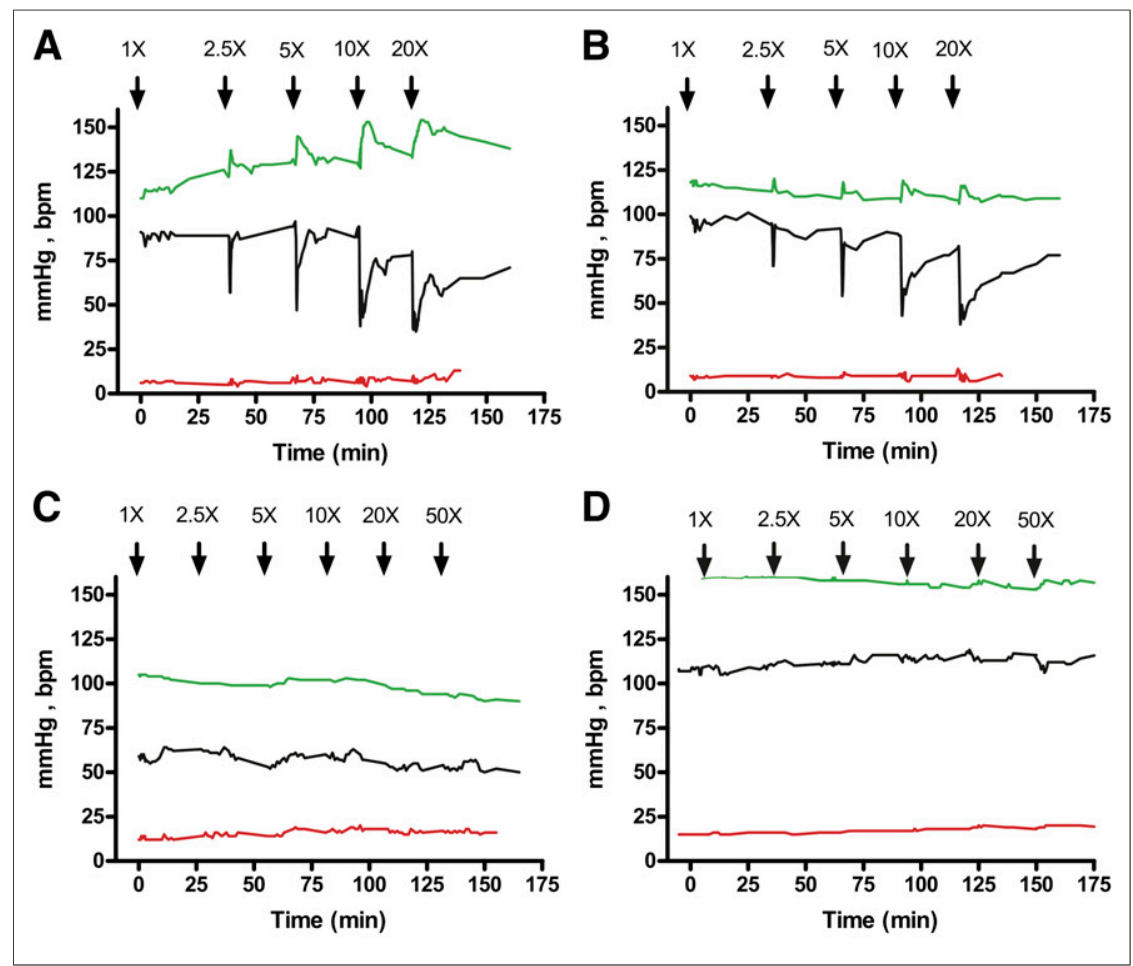

FIGURE 6. Hemodynamic evaluation in dogs of $A M(A)$, agonist $A M(13-52)(B)$, antagonist AM(22-52) (C), and PulmoBind (D). Anesthetized and ventilated mongrel dogs were injected intravenously with cumulative doses of each peptide, and heart rate (green line), mean arterial pressure (black line), and mean pulmonary artery pressure (red line) were monitored. Initial dose (lung scan dose) was maximal dose anticipated for humans (555 MBq; $18.5 \mu \mathrm{g}$ ) in phase 1 clinical study. Animal dose equivalence was computed for body surface area to monitor hemodynamic effects. Each graph depicts typical trace obtained from 2-7 evaluations. bpm $=$ beats per minute.

sidered to be the method of choice for the diagnosis of pulmonary embolism. However, this imaging technique relies on the capture of ${ }^{99 m}$ Tc-MAA in the pulmonary bloodstream causing temporary microembolisms in a sufficient amount to generate a good image (24). If the pulmonary circulation is already compromised, as in patients with severe pulmonary hypertension, this procedure could have deleterious effects; some safety issues have been pointed out for patients with pulmonary hypertension or a right-left shunt (24). Thus, a lung imaging agent such as an AM derivative would expand the clinical relevance of lung scintigraphy because of its ability to image all sizes of vascular beds without any blockade. Moreover, given that AM binding sites are mostly distributed on the pulmonary microvascular endothelium, a molecular imaging agent based on AM could also offer a new diagnostic avenue for pulmonary hypertension because this pathology is associated with microvascular occlusions and endothelial dysfunction not detectable with ${ }^{99 m}$ Tc-MAA (with the exception of chronic thromboembolic pulmonary hypertension).

We demonstrated the relevance of this approach using a monocrotaline-induced pulmonary hypertension rat model (4). However, AM is a biologically active peptide. As a vasodilator, it could be perceived as beneficial, especially if used for the diagnosis of pulmonary hypertension. In fact, AM is recognized as a cardiorenoprotective modulator (25). However, AM also stimulates angiogenesis, a less desirable outcome (26). Although nuclear medicine imaging agents are generally used at a dose devoid of any biologic effect, the safety of clinical procedures is a crucial issue that prompted us to develop a lung imaging agent with antagonist pharmacology.

Many chelators trap ${ }^{99 m} \mathrm{Tc}$; these include diethylenetriaminepentaacetic acid, mercaptoacetyltriglycine, and 6-hydrazinopyridine-3-carboxylic acid. However, a simple tetrapeptide having ${ }^{99 \mathrm{~m}} \mathrm{Tc}$ chelating properties appeared to be more attractive because it was introduced to the peptide chain during synthesis, in accordance with the procedure used for all other amino acids. Incorporation of this peptide chelating moiety greatly improved the labeling yield without any significant difference among the 3 compounds synthesized (Fig. 1). A difference appeared when lung uptake was evaluated. As a matter of fact, the presence of a cyclic structure proved to be crucial for efficient lung uptake because at just 10 min after injection, about $20 \%$ of the linear AM analogs (compounds 1 and 3) remained in the lungs, whereas more than $40 \%$ of AM and the cyclic AM analog (compound 2) were retained in the organ; this activity was maintained even after $30 \mathrm{~min}$ (Fig. 2). These results are in accordance with the results of structure-activity relationship studies of peptides belonging to the calcitonin family. Circular dichroism analyses of AM demonstrated that the peptide structure is composed of $28 \% \alpha$-helix and $18 \% \beta$-sheet. Interestingly, the antagonist $\mathrm{AM}(22-52)$ shares these structural features (14). However, the ring structure composed of 6 residues linked by a disulfide bridge is a characteristic common to all calcitonin peptide family members, despite their low sequence homology, and it has been shown to be important for proper binding and subsequent signaling $(13,26)$. Moreover, amino acid substitution within the ring structure does not seem to markedly alter the biologic activity of the peptide (26). Therefore, combining $\operatorname{AM}(22-52)$ with a flexible ring structure composed of 2 cysteine residues linked by a polyethylene glycol spacer as a substitute for the 6 residues generated a molecule encompassing all of the AM structural features important for ensuring proper receptor binding while avoiding a biologic response. In fact, the use of the polyethylene glycol spacer allowed appropriate spacing without introducing reactive or sensitive chemical groups or

TABLE 2

Absorbed Radiation Dose Estimates for ${ }^{99 m T c-P u l m o B i n d ~}$

\begin{tabular}{lc}
\hline Organs & Radiation dose $(\mathrm{mGy} / \mathrm{MBq})$ \\
\hline Lungs & 0.005 \\
Liver & 0.012 \\
Kidneys & 0.034 \\
Spleen & 0.006 \\
Bladder & 0.010 \\
Testes & 0.002 \\
Ovaries & 0.005 \\
Total body & 0.003 \\
\hline
\end{tabular}


TABLE 3

Comparative Dosimetry of ${ }^{99 m}$ Tc-Radiopharmaceuticals (28)

\begin{tabular}{lclcc}
\hline Radiopharmaceutical & Administered activity (MBq) & Critical organs & Total dose (mSv) & Effective dose (mSv/MBq) \\
\hline 99mTc-PulmoBind & $185-555$ & Kidneys & $1.4-4.2$ & 0.0075 \\
Men & & & $1.7-5.2$ & 0.0094 \\
Women & 185 & Lungs & 2.0 & 0.011 \\
99mTc-MAA & 370 & Bladder & 1.8 & 0.0061 \\
99mTc-DTPA & 370 & Kidneys & 3.3 & 0.0088 \\
99mTc-DMSA & & & \\
& & & \\
DTPA $=$ diethylenetriaminepentaacetic acid; DMSA = dimercaptosuccinic acid. & & \\
\hline
\end{tabular}

increasing too much the hydrophobicity of the molecule. Furthermore, the chelating moiety was added at the $\mathrm{N}$ terminus of the peptide because it was demonstrated that labeling of the peptide at any other position could affect receptor interaction (16).

The results of our binding study correlated with AM structureactivity relationships. Indeed, $A M$ had a $K_{d}$ of $0.17 \pm 0.07 \mathrm{nM}$ for dog lung homogenates (16), and PulmoBind (compound 2) had a $K_{d}$ of $2.6 \pm 0.8 \mathrm{nM}$ for the same preparations (Fig. 5). Given that the same conditions were used to evaluate binding, $\operatorname{AM}(22-52)$ had a binding affinity about 100 times lower than that of AM, and even linear AM (reduced disulfide bridge) had a markedly reduced affinity (6). Thus, adding a cyclic structure to $\operatorname{AM}(22-52)$ to generate PulmoBind enhanced binding significantly. Moreover, the number of accessible binding sites appeared to be higher for PulmoBind than for AM because $B_{\max }$ values were $2,317 \pm 320 \mathrm{fmol} / \mathrm{mg}$ (Fig. 5) and 1,222 $\pm 148 \mathrm{fmol} / \mathrm{mg}(16)$, respectively. This observation can be related to studies of somatostatin, corticotrophin-releasing factor, and bombesin demonstrating that radioactive ligand antagonists label more receptor binding sites than corresponding agonists $(11,27,28)$. Therefore, it appears that the slight loss of binding affinity of PulmoBind is compensated for by a larger number of accessible binding sites, allowing pulmonary circulation imaging similar to that observed with the full-length AM peptide labeled with ${ }^{99 \mathrm{~m}} \mathrm{Tc}$.

As previously mentioned, the AM disulfide ring structure is implicated in proper receptor interaction and the receptor signaling process. Accordingly, the PulmoBind cyclic moiety has

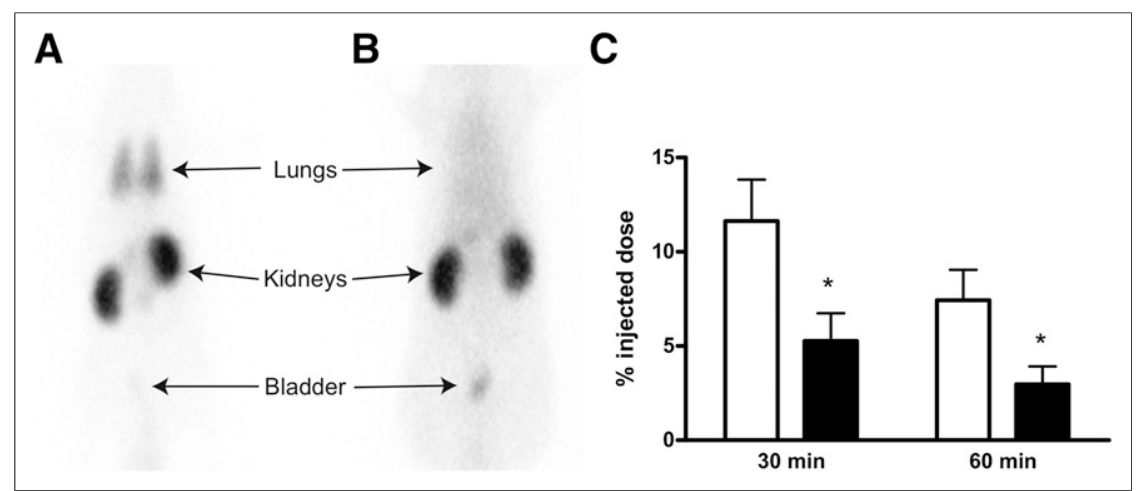

FIGURE 7. Images obtained $30 \mathrm{~min}$ after intravenous injection of ${ }^{99 \mathrm{~m} T c-P u l m o B i n d ~ i n ~}$ vehicle-treated Sprague-Dawley rat $(A)$ and monocrotaline-treated rat (pulmonary arterial hypertension model) (B). (C) Static evaluations of presence of radiotracer in lungs 30 and $60 \mathrm{~min}$ after injection were performed with dual-head $\gamma$-camera and MATLAB software to analyze data from different animals, and significant differences were determined with Student $t$ test. ${ }^{*} P<0.05$. White bars and black bars represent data from vehicle-treated rats and monocrotaline-treated rats, respectively. enhanced receptor binding, but this analog appears to still act as an antagonist, like its parent molecule, as observed in an in vivo hemodynamic evaluation (Fig. 6). In fact, $\operatorname{AM}(22-52)$ did not evoke any changes in MAP, heart rate, or mean pulmonary artery pressure even at 50 times the imaging dose, and similar observations were obtained for PulmoBind; these observations suggest that an AM-based imaging agent such as PulmoBind should not have any acute toxicity effects on the pulmonary vascular system during the course of the examination. In contrast, the truncated $\mathrm{AM}$ analog comprising the ring structure, that is, $\mathrm{AM}(13-52)$, elicited a vasodilation response at only 2.5 times the imaging dose, just like AM. Interestingly, the increase in heart rate was less important with the N-terminally truncated analog, suggesting that this portion of the peptide may be responsible, at least in part, for this specific biologic response.

In the design of targeted imaging compounds, the goal is to obtain high tissue specificity with a high signal-to-noise ratio, appropriate pharmacodynamics, and good pharmacokinetics. Injection of PulmoBind into anesthetized dogs generated a good lung image with low heart and liver uptake, an essential feature for pulmonary vasculature imaging, even at $60 \mathrm{~min}$ after injection (Fig. 4). The lung kinetic profile of PulmoBind was characterized by an uptake plateau, suggesting irreversible binding to its pulmonary receptor. As determined in our in vivo biodistribution study, PulmoBind was retained not only in the pulmonary circulation but also in the kidneys (Fig. 3). As a matter of fact, labeling of the kidneys and bladder was higher than that of other tissues at later time points, with a concomitant decrease in lung, heart, and liver uptake, indicating that the molecule was eliminated mainly through renal excretion. This clearance route was also observed with 99mTc-labeled AM (5). Thus, PulmoBind appears to offer good pulmonary circulation imaging properties, without adverse hemodynamic effects and with urinary elimination, qualities that make it a promising compound for clinical use. However, this elimination route was associated with our finding that the kidneys were the critical organ in terms of radiation dose. Moreover, the close proximity of the urinary tract to radiation-sensitive tissues, such as the reproductive organs, could be a safety concern. However, our dosimetric analysis revealed that the testes and ovaries received the least radiation among the eval- 
uated organs (Table 2), and even the kidneys received lower doses than other critical organs identified with clinically used ${ }^{99 \mathrm{~m}}$ Tc-labeled radiopharmaceuticals (Table 3). Finally, with the proposed injected activity $(185-555 \mathrm{MBq})$, the total effective radiation dose (1.7-5.2 $\mathrm{mSv}$ for a woman) would be comparable to those used in actual nuclear medicine procedures because doses of $0.2-14 \mathrm{mSv}$ are typically used in clinical radiographic examinations (29).

Finally, we demonstrated that radiolabeled PulmoBind could detect a lung microcirculatory perfusion defect associated with pulmonary arterial hypertension using the monocrotaline model. This model causes progressive obliteration of pulmonary vessels, and we demonstrated that it was associated with reduced lung expression of the specific heterodimeric component of the AM receptor, RAMP2 (4). Evidence for the abundant distribution of AM receptors in the pulmonary microcirculation was also obtained previously. A study evaluating the distribution of the calcitonin receptorlike receptor, the other heterodimeric component of the specific AM receptor (CRLR/RAMP2), demonstrated intense staining in the lung capillaries (30). Another study demonstrated colocalization of the endothelial cell marker and the calcitonin receptorlike receptor in pulmonary capillaries and lung endothelial cell expression of the calcitonin receptorlike receptor and RAMP2 (31).

\section{CONCLUSION}

Because nuclear medicine definitely benefits from precise targeting, the development of specific radiotherapeutic drugs and medical imaging agents has attracted much interest, with peptides and proteins forming a large part of the starting material. Many peptides have been used to create new specific radiopharmaceutical compounds, but our work supports studies of somatostatin indicating the usefulness of peptide antagonists as nuclear medicine agents (27,32). PulmoBind, an AM analog, binds AM receptors found at a high density in the pulmonary microvascular endothelium with a high affinity and without causing any adverse hemodynamic response after systemic injection. Once labeled with ${ }^{99 \mathrm{~m}} \mathrm{Tc}$, it provides good pulmonary circulation imaging with a nuclear medicine camera for at least $1 \mathrm{~h}$, and the compound is efficiently eliminated through renal excretion. Thus, PulmoBind labeled with ${ }^{99} \mathrm{~m} \mathrm{Tc}$ is a radiopharmaceutical with the potential to provide new molecular diagnostic modalities for pathologies such as pulmonary hypertension and pulmonary embolism.

\section{DISCLOSURE}

The costs of publication of this article were defrayed in part by the payment of page charges. Therefore, and solely to indicate this fact, this article is hereby marked "advertisement" in accordance with 18 USC section 1734. This work was supported by PulmoScience Inc., the Canadian Institutes of Health Research, and the Montreal Heart Institute Foundation. Jocelyn Dupuis is the scientific director and a shareholder of PulmoScience Inc. No other potential conflict of interest relevant to this article was reported.

\section{REFERENCES}

1. Ruppert A, Lees M, Steinle T. Clinical burden of venous thromboembolism. Curr Med Res Opin. 2010;26:2465-2473.

2. Badesch DB, Champion HC, Sanchez MA, et al. Diagnosis and assessment of pulmonary arterial hypertension. J Am Coll Cardiol. 2009;54(1, suppl):S55-S66.
3. Petersson J, Sanchez-Crespo A, Larsson SA, Mure M. Physiological imaging of the lung: single-photon-emission computed tomography (SPECT). J Appl Physiol. 2007; 102:468-476.

4. Dupuis J, Harel F, Fu Y, et al. Molecular imaging of monocrotaline-induced pulmonary vascular disease with radiolabeled linear adrenomedullin. $\mathrm{J} \mathrm{Nucl} \mathrm{Med}$. 2009;50:1110-1115.

5. Harel F, Fu Y, Nguyen QT, et al. Use of adrenomedullin derivatives for molecular imaging of pulmonary circulation. J Nucl Med. 2008;49:1869-1874.

6. Fu Y, Letourneau M, Nguyen QT, et al. Characterization of the adrenomedullin receptor acting as the target of a new radiopharmaceutical biomolecule for lung imaging. Eur J Pharmacol. 2009;617:118-123.

7. Dupuis J, Caron A, Ruel N. Biodistribution, plasma kinetics and quantification of single-pass pulmonary clearance of adrenomedullin. Clin Sci (Lond). 2005;109: 97-102.

8. Edwards WB, Xu B, Akers W, et al. Agonist-antagonist dilemma in molecular imaging: evaluation of a monomolecular multimodal imaging agent for the somatostatin receptor. Bioconjug Chem. 2008;19:192-200.

9. Brom M, Oyen WJ, Joosten L, et al. ${ }^{68} \mathrm{Ga}$-labelled exendin-3, a new agent for the detection of insulinomas with PET. Eur J Nucl Med Mol Imaging. 2010;37:1345-1355.

10. Reubi JC, Maecke HR. Peptide-based probes for cancer imaging. J Nucl Med. 2008;49:1735-1738.

11. Ginj M, Zhang H, Waser B, et al. Radiolabeled somatostatin receptor antagonists are preferable to agonists for in vivo peptide receptor targeting of tumors. Proc Natl Acad Sci USA. 2006;103:16436-16441.

12. Hamid SA, Baxter GF. Adrenomedullin: regulator of systemic and cardiac homeostasis in acute myocardial infarction. Pharmacol Ther. 2005;105:95-112.

13. Eguchi S, Hirata Y, Iwasaki H, et al. Structure-activity relationship of adrenomedullin, a novel vasodilatory peptide, in cultured rat vascular smooth muscle cells. Endocrinology. 1994;135:2454-2458.

14. Robinson SD, Aitken JF, Bailey RJ, et al. Novel peptide antagonists of adrenomedullin and calcitonin gene-related peptide receptors: identification, pharmacological characterization, and interactions with position 74 in receptor activity-modifying protein 1/3. J Pharmacol Exp Ther. 2009;331:513-521.

15. Champion HC, Nussdorfer GG, Kadowitz PJ. Structure-activity relationships of adrenomedullin in the circulation and adrenal gland. Regul Pept. 1999;85:1-8.

16. Fu Y, Letourneau M, Chatenet D, et al. Characterization of iodinated adrenomedullin derivatives suitable for lung nuclear medicine. Nucl Med Biol. 2011;38:867-874.

17. Boxenbaum H. Interspecies scaling, allometry, physiological time, and the ground plan of pharmacokinetics. J Pharmacokinet Biopharm. 1982;10:201-227.

18. Stabin MG, Siegel JA. Physical models and dose factors for use in internal dose assessment. Health Phys. 2003;85:294-310.

19. Vanbilloen HP, De Roo MJ, Verbruggen AM. Complexes of technetium-99m with tetrapeptides containing one alanyl and three glycyl moieties. Eur J Nucl Med. 1996;23:40-48.

20. Olafsen T, Wu AM. Antibody vectors for imaging. Semin Nucl Med. 2010;40: 167-181.

21. Correia JD, Paulo A, Raposinho PD, Santos I. Radiometallated peptides for molecular imaging and targeted therapy. Dalton Trans. 2011;40:6144-6167.

22. Tweedle MF. Peptide-targeted diagnostics and radiotherapeutics. Acc Chem Res. 2009;42:958-968.

23. Chen K, Conti PS. Target-specific delivery of peptide-based probes for PET imaging. Adv Drug Deliv Rev. 2010;62:1005-1022.

24. Zöphel K, Bacher-Stier C, Pinkert J, Kropp J. Ventilation/perfusion lung scintigraphy: what is still needed? A review considering technetium-99m-labeled macro-aggregates of albumin. Ann Nucl Med. 2009;23:1-16.

25. Bunton DC, Petrie MC, Hillier C, et al. The clinical relevance of adrenomedullin: a promising profile? Pharmacol Ther. 2004;103:179-201.

26. Kuwasako K, Kitamura K, Nagata $S$, et al. Shared and separate functions of the RAMP-based adrenomedullin receptors. Peptides. 2011;32:1540-1550.

27. Cescato R, Maina T, Nock B, et al. Bombesin receptor antagonists may be preferable to agonists for tumor targeting. J Nucl Med. 2008;49:318-326.

28. Perrin MH, Sutton SW, Cervini LA, et al. Comparison of an agonist, urocortin, and an antagonist, astressin, as radioligands for characterization of corticotropinreleasing factor receptors. J Pharmacol Exp Ther. 1999;288:729-734.

29. Mettler FA Jr., Huda W, Yoshizumi TT, Mahesh M. Effective doses in radiology and diagnostic nuclear medicine: a catalog. Radiology. 2008;248:254-263.

30. Hagner S, Stahl U, Knoblauch B, et al. Calcitonin receptor-like receptor: identification and distribution in human peripheral tissues. Cell Tissue Res. 2002;310: 41-50.

31. Hagner S, Haberberger R, Hay DL, et al. Immunohistochemical detection of the calcitonin receptor-like receptor protein in the microvasculature of rat endothelium. Eur J Pharmacol. 2003;481:147-151.

32. Wild D, Fani M, Behe M, et al. First clinical evidence that imaging with somatostatin receptor antagonists is feasible. J Nucl Med. 2011;52:1412-1417. 